\title{
Associação entre comprometimento cognitivo e circunferência da panturrilha em idosos: um estudo transversal
}

\author{
Association between cognitive \\ commitment and calf circumference in \\ elderly: a transversal study
}

\author{
Tainá Soares Risso Rattes ${ }^{1}$ \\ Diana Oliveira Noronha² \\ Manuella Franco Cerqueira da Silva ${ }^{3}$ (1)
}

Emily Rodrigues Mota 4 (1)

Alanna Ribeiro da Silva ${ }^{5}$ (1)

Kionna Oliveira Bernardes Santos ${ }^{6}$

Cleber Luz Santos ${ }^{7}$ (1)

1,3-6Universidade Federal da Bahia (Salvador). Bahia, Brasil. taina.rrattes@gmail.com, manuella.franco@hotmail.com, mily.mota@gmail.com alannaribeiros@gmail.com, kionna.ufba@gmail.com ${ }^{2}$ Centro de Referência Estadual de Atenção à Saúde do Idoso (Salvador). Bahia, Brasil. diananoronhafisio@gmail.com ${ }^{7}$ Autor para correspondência. Universidade Federal da Bahia (Salvador). Bahia, Brasil. cleberluz@ufba.br

RESUMO | INTRODUÇÃO: O Comprometimento cognitivo está correlacionado com circunferência da panturrilha esquerda (CPE) que corresponde a um marcador indireto de sarcopenia. A CPE é uma medida simples e rápida de ser coletada em ambulatórios. Contudo, a magnitude desta correlação parece ser distinta entre os diferentes graus de fragilidade do idoso. OBJETIVO: Verificar a correlação entre a CPE e comprometimento cognitivo nos diferentes níveis de fragilidade do idoso. MATERIAIS E MÉTODOS: Estudo transversal com dados secundários provenientes dos prontuários dos idosos com diagnóstico de demência cadastrados no Centro de Referência Estadual de Atenção à Saúde do Idoso (CREASI). Dados do Mini Exame do estado mental (MEEM) e da CPE foram obtidos da avaliação geriátrica ampla realizada no ingresso ao CREASI. Os dados sociodemográficos dos idosos foram registrados para caracterização da amostra, divididos quanto ao sexo e ao grau de fragilidade. Em cada grupo foi aplicado o teste de Pearson para verificar a correlação entre MEEM e CPE considerando $p$ valor de 0,05. RESULTADOS: Foram avaliados 470 prontuários, 342 mulheres e 128 homens. Os idosos frágeis demonstraram fraca correlação entre os sexos. CONCLUSÃO: Comprometimento cognitivo e CPE estão fracamente correlacionados nos idosos frágeis. O sexo não modificou a correlação entre comprometimento cognitivo e CPE.

PALAVRAS-CHAVE: Disfunção Cognitiva. Envelhecimento. Idoso Fragilizado. Fragilidade. Sarcopenia.
ABSTRACT | BACKGROUND: Cognitive impairment is correlated with left calf circumference (LCC), which corresponds to an indirect marker of sarcopenia. LCC is a simple and quick measure to be collected in outpatient clinics. However, the magnitude of this correlation seems to be different between the different degrees of frailty in the elderly. OBJECTIVE: Verify the correlation between LCC and cognitive impairment at different levels of frailty in the elderly. MATERIALS AND METHODS: Cross-sectional study with secondary data from medical records of elderly people diagnosed with dementia registered at the State Reference Center for Health Care for the Elderly (CREASI). Data were collected from the MiniMental State Examination (MMSE), and the left calf circumference (LCC) was obtained from the comprehensive geriatric assessment carried out on admission of the elderly to CREASI. In addition, the elderly's sociodemographic data were recorded to characterize the sample, divided into gender and degree of frailty. In each group, Pearson's test was applied to verify the correlation between MMSE and LCC, considering a p-value of 0.05 . RESULTS: 470 medical records were evaluated, 342 women and 128 men. The frail elderly showed a weak correlation between the sexes. CONCLUSION: Cognitive impairment and LCC are only weakly correlated in frail older adults. Gender did not modify the correlation between cognitive impairment and LCC.

KEYWORDS: Aging. Cognitive Dysfunction. Frail Elderly. Fragility. Sarcopenia. 


\section{Introdução}

Circunferência da perna esquerda (CPE) é um indicador de perda de massa muscular de fácil coleta em serviços de saúde. A CPE associada a outros indicadores como força muscular e baixa performance física podem indicar quadro de sarcopenia em idosos. ${ }^{1}$ Evidências Científicas apontam que o comprometimento cognitivo (CC) está correlacionado com sarcopenia ${ }^{1}$, alterações emocionais ${ }^{2}$ e fragilidade..$^{3}$ Isto é apoiado pelo fato de que os idosos com diagnóstico de demência comumente apresentam quadros de sarcopenia. ${ }^{4,5}$ Como já há evidências da associação entre sarcopenia e comprometimento cognitivo $0^{6,7}$, é plausível supor que as medidas utilizadas para diagnosticar sarcopenia como CPE, também possuam algum nível de associação com o comprometimento cognitivo. Diante disto, há necessidade de identificar uma possível associação entre CPE e CC para analisar implicações clínicas decorrentes desta possível associação.

Condições clínicas como inflamação sistêmica, estresse oxidativo ${ }^{8}$, resistência à insulina e estilo de vida sedentário parecem reduzir a massa muscular e

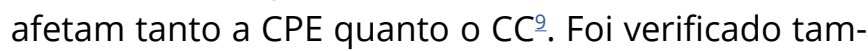
bém que marcadores inflamatórios já foram associados a níveis mais baixos de massa e força muscular e ao desempenho físico ${ }^{10}$ e com quadros de demências, principalmente Alzheimer. ${ }^{11}$ Portanto sujeitos com quadro de demência podem ter CPE reduzida e apresentar associação moderada ou forte com o CC.

A CPE é um procedimento de baixo custo e facilmente replicável em serviços de saúde. ${ }^{13,14}$ Trata-se da medição da circunferência da panturrilha esquerda tendo como pontos de corte $34 \mathrm{~cm}$ para homens e $33 \mathrm{~cm}$ para mulheres. Apesar de não ser recomendado o uso exclusivo desta medida para diagnóstico da sarcopenia, esta medida tem sido utilizada como método de triagem na identificação de uma provável sarcopenia entre os idosos. 12

Assim como a CPE representa uma medida prática e objetiva para triar possíveis casos de sarcopenia, o Mini Exame do Estado Mental (MEEM) corresponde ao teste mais aplicado para identificação do CC. ${ }^{14} \mathrm{O}$ MEEM possui níveis de acurácia internacionalmente aceitos, sendo comumente aplicados em serviços especializados na saúde do idoso. $\frac{15}{}$ Como ambos os testes são realizados durante a Avaliação Geriátrica Ampla (AGA) em serviços de saúde, a análise de possíveis correlações entre elas se torna possível com dados secundários. Estudos prévios relatam a associação entre CC e sarcopenia ${ }^{16,17}$, contudo, não correlacionam esta associação com os diferentes graus de fragilidade. Idosos frágeis com maiores níveis de sarcopenia podem apresentar maiores graus de CC, mas esta afirmação ainda necessita de investigação prévia. Portanto, a associação entre CPE e CC pode ocorrer entre os idosos cadastrados num centro de referência. O objetivo do presente estudo foi verificar a correlação entre a CPE e comprometimento cognitivo nos diferentes níveis de fragilidade do idoso.

\section{Materiais e métodos}

\section{Escopo do estudo}

Trata-se de um estudo observacional, corte transversal de dados secundários dos prontuários de idosos com cadastro ativo no Centro de Referência Estadual de Atenção à Saúde do Idoso (CREASI). A coleta foi realizada de fevereiro a novembro de 2019. Em cada prontuário foi analisada a ficha de avaliação inicial da AGA que é preenchida na admissão do idoso.

Na AGA foram coletados os dados do MEEM que é um instrumento válido para avaliar o comprometimento cognitivo. ${ }^{19}$ O MEEM verifica a orientação, memória e atenção, capacidade de nomeação, obediência a um comando verbal e a escrita ${ }^{20}$ com pontuação de 0 a 30 pontos e tendo o ponto de corte ajustado à escolaridade. ${ }^{21}$ Outra medida coletada na AGA foi à CPE que é um indicador indireto de perda de massa muscular e da sarcopenia em idosos. Foram considerados os valores de ponto de corte de $34 \mathrm{~cm}$ para homens e $33 \mathrm{~cm}$ para mulheres. ${ }^{13,14}$ Conjuntamente foram coletadas variáveis de caracterização da amostra como idade, Índice de massa corporal (IMC), escolaridade, extrato clínico funcional, deambulação, quantidade de medicamentos ingeridos, Timed Up na Go (TUG) e o número de quedas.

Este estudo seguiu as recomendações da resolução 466/12 do Conselho Nacional de Saúde, sendo aprovado pelo comitê de ética em pesquisa em seres humanos da Secretaria da Saúde do Estado da Bahia sob número 2.581.226. O sigilo dos dados, proteção da informação do sujeito de pesquisa, assim como o respeito à privacidade e a individualidade da pessoa idosa foram garantidos através da restrição de acessos a base de dados aos pesquisadores principais. 
O uso dos dados para análise não continha informações que identificassem os sujeitos.

\section{Amostra}

Participaram deste estudo os idosos com diagnóstico de demência registrada na AGA. Os níveis de fragilidade foram identificados pelo índice de vulnerabilidade clínico funcional (IVCF-20) proposto por Moraes et al., 2016.22 O IVCF-20 foi desenvolvido para identificar rapidamente os idosos frágeis. Trata-se de um questionário que contempla aspectos multidimensionais da saúde do idoso, sendo composto por 20 questões relacionadas à idade, autopercepção de saúde, incapacidades funcionais, cognição, humor, mobilidade, capacidade aeróbica, marcha, incontinência esfincteriana, comunicação e comorbidades múltiplas. O resultado do IVCF-20 é expresso na escala de 1 a 10, sendo considerado idosos robusto ( 1 a 3 ), idosos em risco de fragilização (4 e 5) e idoso frágil (6 a 10). $\underline{22}$

\section{Procedimentos}

A coleta foi realizada por 13 pesquisadores colaboradores distribuídos em cinco turnos de coleta. Todos receberam treinamento prévio e realizaram a coleta através de formulário eletrônico no computador. Para minimizar as coletas duplicadas e o retrabalho da equipe, foi inserido um pesquisador supervisor que realizava revisões semanais na base de dados.

A coleta foi realizada numa sala restrita do Núcleo de Ensino e Pesquisa (NEP) do CREASI. Ambiente devidamente equipado para esta finalidade com quatro computadores disponíveis. Dúvida sobre dados contidos no prontuário podiam ser discutidas com os pesquisadores responsáveis que conheciam com profundidade a AGA. Os dados coletados foram armazenados eletronicamente numa pasta virtual do NEP. A base de dados foi revisada por um pesquisador externo para verificar inconsistências e erros de coleta para posteriormente ser liberada para análise dos dados. Portanto, a base de dados foi considerada segura e confiável para análise estatística. Uma cópia registrada com dados de identificação dos idosos substituídos por códigos alfanuméricos foi liberada para análise estatística.

\section{Análise dos dados}

Foi realizada uma análise descritiva dos dados clínicos e funcionais para caracterização da amostra.
Para caracterização da amostra neste estudo foi realizada uma análise descritiva dos dados de identificação dos sujeitos. Dados quantitativos foram apresentados com medidas de tendência centrais (médias ou medianas) e suas medidas dispersivas. As medidas ordinárias foram apresentadas como frequências. $\mathrm{O}$ teste de Kolmogorov-Smirnov foi aplicado para verificar a adesão à curva de normalidade, considerando $p \leq 0,05$. Os idosos foram divididos em 2 grupos: 1 ) Idosas frágeis, 2) Idosos muito frágeis, 3) Idosas em risco de fragilização e 4) Idosos em risco de fragilização. A correlação entre MEEM e CPE através do teste de Pearson foi realizada em cada grupo separadamente considerando $p \leq 0,05$. As análises estatísticas foram realizadas no software Statistical Package for the Social Sciences versão 20.0 para Windows (SPSS Inc, Chicago, IL, EUA).

\section{Resultados}

Foram identificados 3282 prontuários no CREASI, sendo que 1092 apresentavam algum tipo de demência. Após aplicação dos critérios de exclusão foram selecionados 470 idosos, sendo 342 mulheres e 128 homens distribuídos nos quatro grupos do estudo composto por 22 homens em risco de fragilização, 106 homens frágeis, 44 mulheres em risco de fragilização e 298 muIheres frágeis (Figura 1). A maior parte dos idosos nos quatro grupos possuía de 1 a 7 anos de estudo com deambulação livre e eram semelhantes quanto à idade $(F=2,605 ; p=0,101)$ e ao IMC $(F=1,191 ; p=0,206)$. O número de quedas no último ano foi semelhante entre os quatro grupos ( $F=0.577 ; p=0,448$ ) e a quantidade de medicamentos em consumo diferiu entre os grupos $(F=6,507 ; p=0,011)$ sendo maior entre as mulheres frágeis. Foi observado também que o tempo médio do TUG diferiu entre os grupos $(F=3,302 ; p=0,051)$ sendo maior nos indivíduos frágeis, independente do sexo. Os demais dados que caracterizam os indivíduos do estudo estão descritos na Tabela 1.

A correlação entre MEEM e CPE foi analisada em cada grupo e indicou baixo grau de correlação em todos. Contudo, a correlação foi significativa nos grupos de idosos frágeis. As mulheres em risco de fragilização apresentaram o menor grau de correlação sem significância estatística $(r=-0,115, p=0,458)$ e os homens frágeis apresentaram maior índice de correlação ( $r=$ $0,345, p=0,000)$. A distribuição das correlações em cada grupo está descrita na Figura 2. 
Tabela 1. Características dos idosos com demência do Centro de Referência Estadual a Atenção do Idoso em Salvador, Bahia

\begin{tabular}{|c|c|c|c|c|c|c|}
\hline & \multicolumn{2}{|c|}{ Risco de Fragilização } & \multicolumn{2}{|l|}{ Frágeis } & \multirow[b]{2}{*}{ Test T } & \multirow[b]{2}{*}{$\mathrm{p}$} \\
\hline & $\begin{array}{l}\text { Homens } \\
(n=22)\end{array}$ & $\begin{array}{l}\text { Mulheres }(n= \\
\text { 44) }\end{array}$ & $\begin{array}{l}\text { Homens } \\
(n=106)\end{array}$ & $\begin{array}{l}\text { Mulheres } \\
(n=298)\end{array}$ & & \\
\hline Idade (média $\pm D P$ ) & $76,6 \pm 8,77$ & $74,5 \pm 6,97$ & $76,2 \pm 11,33$ & $79,2 \pm 8,29$ & 0,567 & 0,330 \\
\hline \multicolumn{7}{|l|}{ Escolaridade (n (\%)) } \\
\hline Analfabeto & $1(4,8)$ & $15(34,9)$ & $21(17,9)$ & $88(26,8)$ & & \\
\hline 1 a 7 anos & $13(61,9)$ & $19(44,2)$ & $64(54,7)$ & $176(53,7)$ & & \\
\hline 8 anos ou mais & $7(33,3)$ & $9(20,9)$ & $32(27,4)$ & $64(19,5)$ & & \\
\hline IMC (média $\pm D P$ ) & $24,4 \pm 3,7$ & $25,5 \pm 5,3$ & $24,5 \pm 3,5$ & $24,9 \pm 5,1$ & 1,56 & 0,089 \\
\hline $\begin{array}{l}\text { Quantidade de } \\
\text { medicamentos } \\
\text { (média } \pm D P \text { ) }\end{array}$ & $4,8 \pm 2,8$ & $4,8 \pm 3,1$ & $4,38 \pm 2,6$ & $5,2 \pm 3,0$ & 1,09 & 0,13 \\
\hline $\begin{array}{l}\text { Extrato Clínico Funcional } \\
\text { (média } \pm D P)\end{array}$ & $4,8 \pm 0,91$ & $5,2 \pm 1,1$ & $7,8 \pm 1,1$ & $7,6 \pm 1,1$ & 0,893 & 0,243 \\
\hline $\begin{array}{l}\text { Panturrilha Esquerda } \\
\text { (média } \pm D P \text { ) }\end{array}$ & $34,47 \pm 3,4$ & $34,1 \pm 3,5$ & $33,4 \pm 3,6$ & $32,4 \pm 3,8$ & 1,752 & 0,092 \\
\hline $\begin{array}{l}\text { MiniMental } \\
(\text { média } \pm D P)\end{array}$ & $20,0 \pm 6,2$ & $16,3 \pm 6,3$ & $14,1 \pm 6,6$ & $13,0 \pm 6,1$ & 0,374 & 0,879 \\
\hline \multicolumn{7}{|l|}{ Deambulação (n (\%)) } \\
\hline Livre & $15(71,4)$ & $31(72,1)$ & $60(51,3)$ & $194(59,1)$ & & \\
\hline Bengala & $1(4,8)$ & $3(7)$ & $4(3,4)$ & $18(5,5)$ & & \\
\hline Auxiliado por Terceiros & $O(0)$ & $1(2,3)$ & $6(5,1)$ & $14(4,3)$ & & \\
\hline Cadeira de Rodas & $O(0)$ & $1(2,3)$ & $14(12,0)$ & $31(9,5)$ & & \\
\hline Muleta & $1(4,8)$ & $0(0)$ & $4(3,4)$ & $1(0,3)$ & & \\
\hline Andador & $O(0)$ & $O(0)$ & $1(0,9)$ & $6(1,8)$ & & \\
\hline Outros & $0(0)$ & $0(0)$ & $2(1,7)$ & $3(0,9)$ & & \\
\hline Não preenchido & $4(19,0)$ & $7(16,3)$ & $7(16,3)$ & $61(18,6)$ & & \\
\hline TUG (média $\pm D P$ ) & $10,2 \pm 1,58$ & $13,4 \pm 4,36$ & $21,5 \pm 24,69$ & $18,8 \pm 10,9$ & 1,273 & 0,845 \\
\hline $\begin{array}{l}\text { Número Quedas no último } \\
\text { ano } \\
\text { (média } \pm D P)\end{array}$ & $0,55 \pm 0,98$ & $1,0 \pm 1,8$ & $0,8 \pm 1,6$ & $1,0 \pm 1,6$ & 0,324 & 0,754 \\
\hline
\end{tabular}

Figura 1. Fluxograma de seleção dos prontuários de idosos com cadastros ativos no CREASI

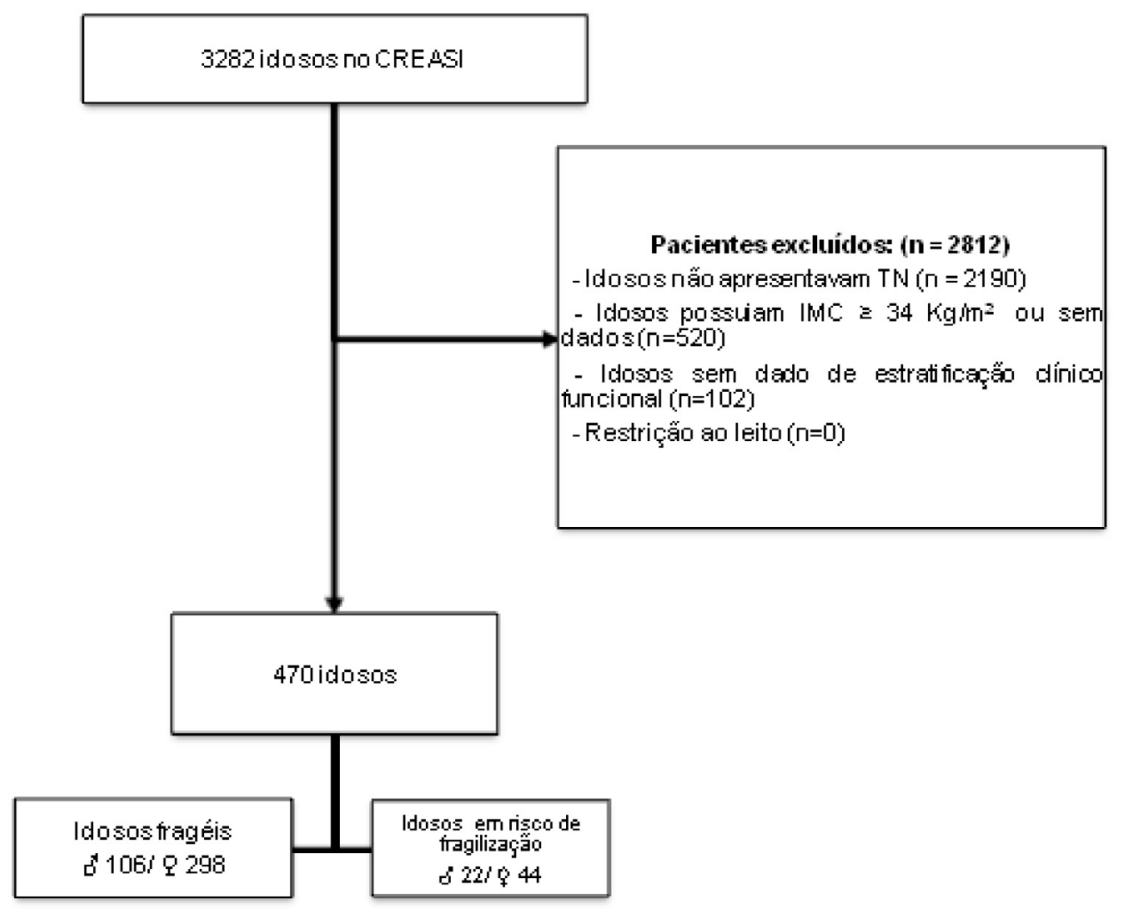


Figura 2. Correlação entre Mini mental e circunferência da panturrilha esquerda (cm) de acordo com o sexo e extrato clínico-funcional. A figura $2 A$ corresponde a idosos frágeis com correlação de $r=0,345$ e $p=0,000 *$, a figura 2B corresponde a idosos em risco de fragilização com correlação de $r=0,286$ e $\mathbf{p}=0,209$. A figura $2 C$ mostra a correlação nas idosas frágeis tendo $r=0,244$ e $p=0,000$ * e a figura 2D traz a correlação de idosas em risco de fragilização sendo $r=-0,115$ e $p=0,458$

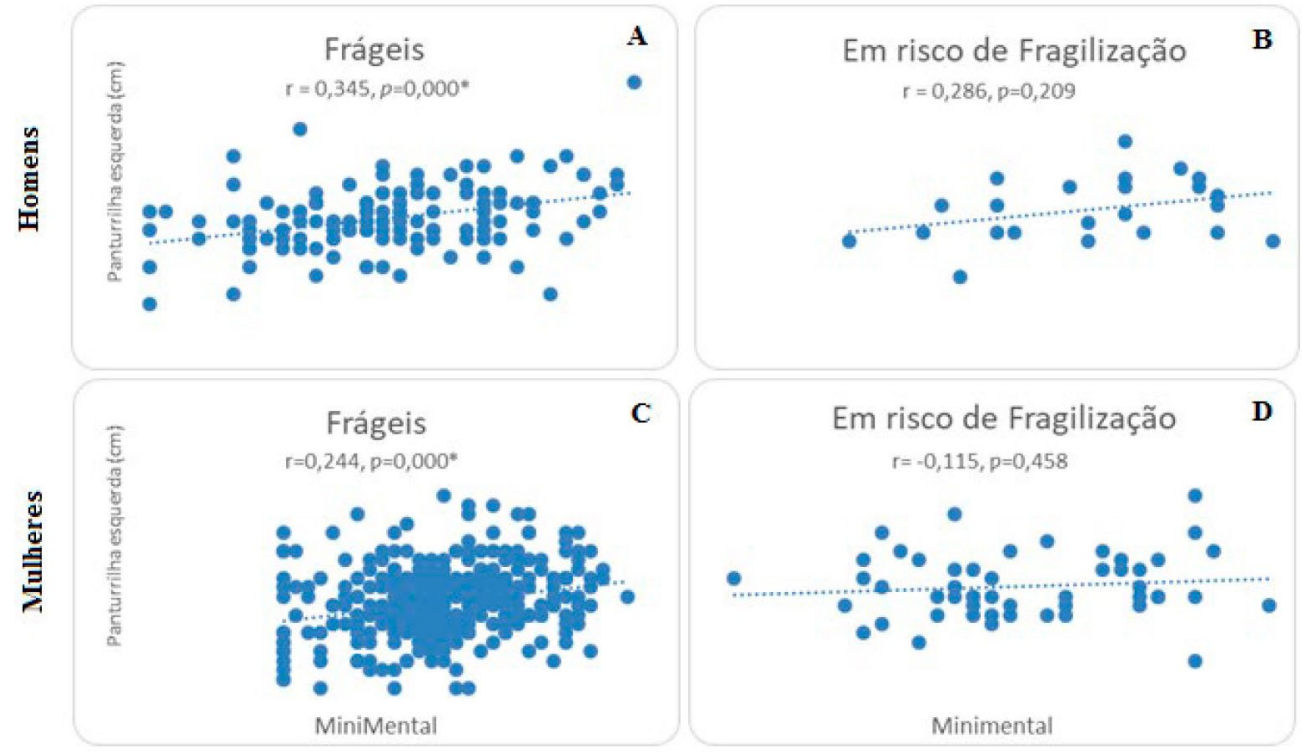

\section{Discussão}

A circunferência da panturrilha esquerda apresentou uma fraca correlação com o comprometimento cognitivo em idosos frágeis independente do sexo. Embora revisões sistemáticas apontem uma correlação de moderada a forte, entre estas variáveis ${ }^{23}$, o presente estudo evidenciou fraca correlação apenas nos idosos frágeis. Diferentes estudos afirmam que sexo feminino tende a apresentar maior magnitude desta correlação. ${ }^{1} \mathrm{~A}$ justificativa corresponde a tendência do sexo feminino apresentar mais déficits neurológicos e menor desempenho físico quando comparado ao sexo masculino. Estes achados não foram reproduzidos no presente estudo relevando que o sexo não modificou a magnitude da correlação entre MEEM e CPE.

Apesar disto, a sarcopenia esteve correlacionada ao comprometimento cognitivo e aos sintomas depressivos em homens idosos que moram em comunidade ${ }^{24}$, assim como esta correlação também foi evidenciada quando foram utilizados diferentes parâmetros de avaliação da sarcopenia. ${ }^{25}$ Contudo, o presente estudo foi um dos poucos que utilizou o CPE para correlacionar com MEEM. Este fato não inviabiliza a análise, mas permite análises que justifiquem a divergência dos achados.

Apesar da literatura demonstrar de forma robusta a associação entre sarcopenia e comprometimento cognitivo nossos achados contradizem as principais revisões sistemáticas sobre este assunto, e demonstra que o sexo não influencia essa correlação. 1,26,27 A justificativa para analisar separadamente por sexo reside na identificação dos fatores de risco à sarcopenia inerentes ao homem e à mulher. Portanto, compreender o papel do sexo nesta correlação permite avançar em estudos sobre os fatores fisiológicos, comportamentais e sociais adotados por homens ou mulheres idosas.

A inclusão da CPE no presente estudo se deve a facilidade do uso clínico, o baixo custo e a praticidade na coleta da informação. Trata-se de uma medida indireta considerada válida para monitorar a sarcopenia., ${ }^{13,14} \mathrm{~A}$ circunferência da panturrilha pode ser influenciada pela presença de tecido adiposo e por este motivo foram excluídos os pacientes com IMC superior a $34 \mathrm{~kg} / \mathrm{m} 2$. 
A fragilidade é o fator agregado que induz maiores níveis de fraqueza muscular em idosos de ambos os sexos. ${ }^{23}$ O presente estudo evidenciou maior correlação entre a CPE e o MEEM em idosos frágeis demonstrando que a fragilidade aumenta as chances desta correlação ocorrer. A importância do diagnóstico precoce da fragilidade reside na possibilidade de prevenir incapacidades e fornecer oportunidades para os idosos permanecerem saudáveis e independentes mais tempo. 23,26

Os resultados encontrados neste estudo não apoiam as descobertas pregressas de forte associação entre sarcopenia e comprometimento cognitivo $0^{16,29,30}$, mas suportam a ideia de que há uma relação entre estas variáveis. O desenho metodológico transversal adotado neste estudo difere dos artigos prospectivos inclusos nas revisões sistemáticas. ${ }^{31}$ Apesar disto, os desenhos metodológicos distintos não impediram a análise comparativa entre os estudos. O presente estudo possui dados robustos coletados por médicos geriatras num centro de referência especializado em saúde do idoso, assegurando a qualidade da informação coletada. Conjuntamente, o tamanho amostral é suficiente para garantir poder de estudo para as análises estatísticas implementadas.

A CPE juntamente com a medida de força muscular e a capacidade física devem ser utilizadas em serviços de saúde para identificação de sarcopenia, mas extrapolar o uso da CPE para investigar alterações do comprometimento cognitivo deve ser visto com cautela. Novos estudos prospectivos que abordem a relação entre CPE e CC podem ajudar a entender com profundidade esta relação, cujo estudo apresentou indícios de sua existência. A relação entre estas medidas apresenta-se incipiente para uso clínico, sendo necessário avançar na investigar juntamente com os mecanismos fisiopatológicos que embasam esta associação com mais robustez.

O presente estudo apresenta como limitações o uso de dados secundários que foram registrados por profissionais de saúde durante as suas atividades laborais. Tais dados podem conter viés de aferição que não foram identificados pelos pesquisadores. Para minimizar isto foram inclusos uma amostra elevada, capaz de amenizar este viés. A ausência de informações mais detalhadas sobre a sarcopenia impediram a correlação direta entre sarcopenia e CC. Por este motivo foi feita a análise apenas com o CPE.

\section{Conclusão}

O CC e a CPE estão fracamente correlacionados apenas nos idosos frágeis de ambos os sexos. Esta associação não foi encontrada nos Idosos em risco de fragilização. A fraca correlação entre CPE e CC indicam que essas medidas juntas possuem baixa relevância clínica, não sendo possível afirmar que alterações na CPE influenciarão o CC mesmo em idosos frágeis.

\section{Agradecimentos}

A equipe de pesquisadores agradece aos funcionários do Centro de Referência Estadual de Atenção à Saúde do Idoso (CREASI) pelo apoio durante a fase de coleta de dados, em especial à diretora Dra Mônica Frank e a vice-coordenadora a Dra Renata Caires pelo acesso aos prontuários e uso da infraestrutura da sala do Núcleo de ensino e Pesquisa da instituição.

\section{Contribuições dos autores}

Rattes TSR participou da coleta de dados da pesquisa, interpretação de dados, concepção, delineamento, análise estatística dos dados da pesquisa, e redação do artigo cientifico. Noronha DO participou do recrutamento de participantes, planejamento do projeto de pesquisa e delineamento. Silva MFC, Silva AR e Mota ER participaram da coleta de dados do estudo. Santos KOB participou do planejamento do projeto de pesquisa e delineamento. Luz-Santos C participou do recrutamento de participantes, planejamento do projeto de pesquisa, concepção, delineamento, análise estatística dos dados da pesquisa e redação do artigo cientifico.

\section{Conflitos de interesses}

Nenhum conflito financeiro, legal ou político envolvendo terceiros (governo, empresas e fundações privadas, etc.) foi declarado para nenhum aspecto do trabalho submetido (incluindo, mas não se limitando a subvenções e financiamentos, participação em conselho consultivo, desenho de estudo, preparação de manuscrito, análise estatística, etc.).

\section{Referências}

1. Cipolli GC, Yassuda MS, Aprahamian I. Sarcopenia Is Associated with Cognitive Impairment in Older Adults: A Systematic Review and Meta-Analysis. J Nutr Health Aging. 2019;23(6):525-31. https:// doi.org/10.1007/s12603-019-1188-8

2. Silva JNMA, Leite MT, Gaviraghi LC, Kirsten VR, Kinalski SS, Hildebrandt LM, et al. Dimensões preditoras das condições clínicofuncionais e cognição em idosos. Rev. Bras. Enferm. 2020;73(Suppl 3):e20190162. https://doi.org/10.1590/0034-7167-2019-0162 
3. Chaves AS, Santos AM, Alves MTSSB, Salgado FN. Associação entre declínio cognitivo e qualidade de vida de idosos hipertensos. Rev. bras. geriatr. Gerontol. 2015;18(3):545-56. https://doi.org/10.1590/1809-9823.2015.14043

4. Yu R, Morley JE, Kwok T, Leung J, Cheung O, Woo J. The Effects of Combinations of Cognitive Impairment and Pre-frailty on Adverse Outcomes from a Prospective Community-Based Cohort Study of Older Chinese People. Front Med. 2018;5:50. https://doi. org/10.3389/fmed.2018.00050

5. Pacifico J, Geerlings MAJ, Reijnierse EM, Phassouliotis C, Lim WK, Maier AB. Prevalence of sarcopenia as a comorbid disease: A systematic review and meta-analysis. Exp Gerontol. 2020;131:110801. https://doi.org/10.1016/j.exger.2019.110801

6. Ida S, Nakai M, Ito S, Ishihara Y, Imataka K, Uchida A, et al. Association Between Sarcopenia and Mild Cognitive Impairment Using the Japanese Version of the SARC-F in Elderly Patients With Diabetes. J Am Med Dir Assoc. 2017;18(9):809.e9-809.e13. https:// doi.org/10.1016/j.jamda.2017.06.012

7. Ishii H, Marizako H, Doi T, Tsutsumimoto K, Shimada H. Associations of skeletal muscle mass, lower-extremity functioning, and cognitive impairment in community-dwelling older people in Japan. J Nutr Health Aging. 2018;23(1):35-45. https://doi. org/10.1007/s12603-018-1110-9

8. Szlejf C, Suemoto CK, Lotufo PA, Benseñor IM. Association of Sarcopenia With Performance on Multiple Cognitive Domains: Results From the ELSA-Brasil Study. J Gerontol A Biol Sci Med Sci. 2019;74(11):1805-11. https://doi.org/10.1093/gerona/glz118

9. Leite LEA, Resende TL, Nogueira GM, Cruz IBM, Schneider $\mathrm{RH}$, Gottlieb MGV. Envelhecimento, estresse oxidativo e sarcopenia: uma abordagem sistêmica. Rev. bras. geriatr. gerontol. 2012;15(2):365-80. https://doi.org/10.1590/S180998232012000200018

10. Van Alphen HJM, Volkers KM, Blankevoort CG, Scherder EJA, Hortobágyi T, van Heuvelen MJG. Older Adults with Dementia Are Sedentary for Most of the Day. PLoS ONE. 2016;11(3):e0152457. https://doi.org/10.1371/journal.pone.0152457

11. Van Atteveld VA, Van Ancum JM, Reijnierse EM, Trappenburg MC, Meskers CGM, Maier AB. Erythrocyte sedimentation rate and albumin as markers of inflammation are associated with measures of sarcopenia: a cross-sectional study. BMC Geriatr. 2019;19(1):233. https://doi.org/10.1186/s12877-019-1253-5

12. Franceschi C, Campisi J. Chronic Inflammation (Inflammaging) and Its Potential Contribution to Age-Associated Diseases. J Gerontol A Biol Sci Med Sci. 2014;69 (1):S4-9. https://doi. org/10.1093/gerona/glu057

13. Pagotto V, Santos KF, Malaquias SG, Bachion MM, Silveira EA. Circunferência da panturrilha: validação clínica para avaliação de massa muscular em idosos. Rev Bras Enferm. 2018;71(2):322-8. https://doi.org/10.1590/0034-7167-2017-0121
14. Kawakami R, Murakami H, Sanada K, Tanaka N, Sawada SS, Tabata I, et al. Calf circumference as a surrogate marker of muscle mass for diagnosing sarcopenia in Japanese men and women. Geriatr Gerontol Int. 2015;15(8):969-76. https://doi.org/10.1111/ ggi.12377

15. Almeida OP. Mini exame do estado mental e o diagnóstico de demência no Brasil. Arq. Neuro-Psiquiatr. 1998;56(3B):605-12. https://doi.org/10.1590/S0004-282X1998000400014

16. Mitchell AJ. A meta-analysis of the accuracy of the mini-mental state examination in the detection of dementia and mild cognitive impairment. J Psychiatr Res. 2009;43(4);411-31. https://doi. org/10.1016/j.jpsychires.2008.04.014

17. Chang K, Hsu TH, Wu WT, Huang KC, Han DS. Association between sarcopenia and cognitive impairment: A systematic review and meta-analysis. J Am Med Dir Assoc. 2016:17(12):1164. e7-1164.e15. https://doi.org/10.1016/j.jamda.2016.09.013

18. Maeda K, Akagi J. Cognitive impairment is independently associated with definitive and possible sarcopenia in hospitalized older adults: The prevalence and impact of comorbidities. Geriatr Gerontol Int. 2017;17(7):1048-56. https://doi.org/10.1111/ ggi.12825

19. Wibelinger LM, Jorge MSG, Portella MR, Doring M, Avan BS, Frâncio F. Síndrome da Fragilidade em idosos institucionalizados. Revista Interdisciplinar de estudos em Saúde da UNIARP. 2020;9(1):19. https://doi.org/10.33362/ries.v9i1.1745

20. Folstein MF, Folstein SE, Mchugh PR. Mini-mental state. A practical method for grading the cognitive state of patients for the clinician. J Psychiatr Res 1975;12(3):189-98. https://doi. org/10.1016/0022-3956(75)90026-6

21. Melo DM, Barbosa AJG. O uso do Mini-Exame do Estado Mental em pesquisas com idosos no Brasil: uma revisão sistemática. Ciênc. saúde coletiva. 2015;20(12):3865-76. https:// doi.org/10.1590/1413-812320152012.06032015

22. Moraes END, Carmo JAD, Moraes FLD, Azevedo RS, Machado CJ, Montilla DER. Índice de Vulnerabilidade Clínico Funcional-20 (IVCF-20): reconhecimento rápido do idoso frágil. Rev Saúde Pública. 2016;50(81). https://doi.org/10.1590/S1518$\underline{8787.2016050006963}$

23. Ohta Y, Nomura E, Hatanaka N, Osakada Y, Matsumoto N, Sasaki R, et al. Female dominant association of sarcopenia and physical frailty in mild cognitive impairment and Alzheimer's disease. J Clin Neurosci. 2019;70:96-101. https://doi.org/10.1016/j. jocn.2019.08.062

24. Kim M, Won CW. Sarcopenia Is Associated with Cognitive Impairment Mainly Due to Slow Gait Speed: Results from the Korean Frailty and Aging Cohort Study (KFACS). Int. J. Environ. Res. Public Health. 2019;16(9):1491. https://doi.org/10.3390/ ijerph16091491 
25. Rodríguez-Rejón Al, Artacho R, Ruiz-López MD.

Anthropometric Measurements and Cognitive Impairment Rather Than Nutrition Status Are Associated With Sarcopenia in LongTerm Care Residents. utr Clin Pract. 2020;35(4):642-8. https://doi. org/10.1002/ncp.10370

26. Xu W, Chen T, Shan Q, Hu B, Zhao M, Deng X, et al. Sarcopenia Is Associated with Cognitive Decline and Falls but Not Hospitalization in Community- Dwelling Oldest Old in China: A Cross-Sectional Study. Medical Sci Monit. 2020;26:e919894. https://doi.org/10.12659/msm.919894

27. Peng TC, Chen WL, Wu LW, Chang YW, Kao TW. Sarcopenia and cognitive impairment: A systematic review and metaanalysis. Clin Nutr. 2020;39(9):2695-701. https://doi.org/10.1016/j. clnu.2019.12.014

28. Hsu YH, Liang CK, Chou MY, Liao MC, Lin YT, Chen LK, et al. Association of cognitive impairment, depressive symptoms and sarcopenia among healthy older men in the veteran's retirement community in southern Taiwan: a cross-sectional study. Geriatr Gerontol Int. 2014;14(Suppl 1):102-8. https://doi.org/10.1111/ ggi.12221
29. Abellan Van Kan G, Cesari M, Gillette-Guyonnet S, Dupuy C, Nourhashémi F, Schott AM, et al. Sarcopenia and cognitive impairment in elderly women: results from the EPIDOS cohort. Age Ageing. 2013;42(2):196-202. https://doi.org/10.1093/ageing/ afs173

30. Tay L, Ding Y Y, Leung BP, Ismail NH, Yeo A, Tay KS, et al. Sex-specific differences in risk factors for sarcopenia amongst community-dwelling older adults. Age. 2015;37(6):121. https://doi. org/10.1007/s11357-015-9860-3

31. Mckee A, Morley JE. Hormones and sarcopenia. Curr. Opin. Endocr. Metab. Res. 2019;9:34-9. https://doi.org/10.1016/j. coemr.2019.06.006 